\title{
Collecting duct-specific knockout of endothelin-1 causes hypertension and sodium retention
}

\author{
Dowhan Ahn, ${ }^{1,2}$ Yuqiang Ge, ${ }^{1}$ Peter K. Stricklett, ${ }^{1}$ Pritmohinder Gill, ${ }^{1}$ Deborah Taylor, ${ }^{1}$ \\ Alisa K. Hughes, ${ }^{1}$ Masashi Yanagisawa, ${ }^{3}$ Lance Miller, ${ }^{1}$ Raoul D. Nelson, ${ }^{1}$ and Donald E. Kohan ${ }^{1,4}$ \\ ${ }^{1}$ Division of Nephrology, University of Utah Health Sciences Center, Salt Lake City, Utah, USA. \\ 2Department of Physiology, Kosin University College of Medicine, Pusan, South Korea. ${ }^{3}$ Howard Hughes Medical Institute, \\ University of Texas Southwestern, Dallas, Texas, USA. ${ }^{4}$ Salt Lake Veterans Affairs Medical Center, Salt Lake City, Utah, USA.
}

\begin{abstract}
In vitro studies suggest that collecting duct-derived (CD-derived) endothelin-1 (ET-1) can regulate renal Na reabsorption; however, the physiologic role of CD-derived ET-1 is unknown. Consequently, the physiologic effect of selective disruption of the ET-1 gene in the CD of mice was determined. Mice heterozygous for aquaporin 2 promoter Cre recombinase and homozygous for loxP-flanked exon 2 of the ET-1 gene (called CD-specific KO of ET-1 [CD ET-1 KO] mice) were generated. These animals had no CD ET-1 mRNA and had reduced urinary ET-1 excretion. CD ET-1 KO mice on a normal Na diet were hypertensive, while body weight, $\mathrm{Na}$ excretion, urinary aldosterone excretion, and plasma renin activity were unchanged. CD ET-1 KO mice on a high-Na diet had worsened hypertension, reduced urinary $\mathrm{Na}$ excretion, and excessive weight gain, but showed no differences between aldosterone excretion and plasma renin activity. Amiloride or furosemide reduced BP in CD ET-1 KO mice on a normal or high-Na diet and prevented excessive Na retention in salt-loaded CD ET-1 KO mice. These studies indicate that CD-derived ET-1 is an important physiologic regulator of renal $\mathrm{Na}$ excretion and systemic BP.
\end{abstract}

\section{Introduction}

Endothelin-1 (ET-1) was initially described as a potent endothelial cell-derived vasoconstrictor (1); however, the peptide is now known to be produced by many cell types and to elicit multiple biologic effects (2). The kidney is likely an important target; ET-1 causes renal vasoconstriction, mesangial cell contraction, glomerular cell proliferation, ECM accumulation, and alterations in nephron fluid and electrolyte transport (2). While many renal cell types synthesize and bind ET-1, the collecting duct (CD) is of particular importance: The renal inner medulla contains the highest concentration of ET-1 in the body (3), and the inner medullary CD (IMCD) is the predominant renal site of ET-1 production (4-8) and receptor expression (9-11).

In vitro studies suggest that ET-1 inhibits $\mathrm{Na}$ and water reabsorption in the cortical $\mathrm{CD}(\mathrm{CCD})$ and IMCD and that this occurs through activation of the ET B receptor (ETRB). ET-1 inhibits vasopressin-stimulated (AVP-stimulated) water flux in isolated CCD $(9,12)$ and reduces AVP-stimulated cyclic AMP accumulation $(13-15)$ and osmotic water permeability $(16,17)$ in isolated IMCDs. ET-1 also inhibits mineralocorticoid and AVP-stimulated $\mathrm{Na}$ and $\mathrm{Cl}$ reabsorption in isolated CCDs $(12,18,19)$ and decreases $\mathrm{Na} / \mathrm{K}$-ATPase activity in suspensions of IMCDs (20). Despite these data, demonstrating such an ET-1 effect in vivo and clarifying how CD-derived ET-1 physiologically regulates $\mathrm{Na}$ and water transport has been problematic. This difficulty stems, in part, from

Nonstandard abbreviations used: aquaporin2 (AQP2); collecting duct (CD); cortical CD (CCD); digitonin (DIG); endothelin (ET); epithelial Na channel (ENaC); ET B receptor (ETRB); inner medullary $\mathrm{CD}$ (IMCD); medullary blood flow (MBF); plasma renin activity (PRA); vasopressin (AVP).

Conflict of interest: The authors have declared that no conflict of interest exists.

Citation for this article: J. Clin. Invest. 114:504-511 (2004)

doi:10.1172/JCI200421064 the inability to discriminate between ET-1 effects on the nephron as opposed to the vasculature. Administration of ET agonists or antagonists typically alters renal plasma flow and glomerular filtration rate, effects that can impact urinary $\mathrm{Na}$ and water excretion (2). Even when ET has been given systemically in doses titrated to not detectably alter renal hemodynamics, increased $\mathrm{Na}$ excretion is observed in some $(21,22)$, but not all (23-26), studies. A number of studies have determined that systemically administered ET increases water excretion, even in the absence of any increase in $\mathrm{Na}$ excretion (23-25). Taken together, these studies suggest that ET-1 may inhibit nephron sodium and water reabsorption. It is unclear, however, if CD-derived ET-1 does in fact regulate $\mathrm{Na}$ and water reabsorption. Furthermore, there is no agreement on what physiologic or pathophysiologic roles the peptide serves in modulating renal salt and water excretion.

Gene-targeting strategies might permit more specific evaluation of ET-1-regulated $\mathrm{Na}$ and water excretion. Unfortunately, every mouse homozygous for $\mathrm{KO}$ of a component of the ET system (ET-1, ET-2, ET-3, ET receptor A, ETRB) has a lethal phenotype (27-31). Of greater utility are studies using spotted lethal rats that carry a natural mutation in the ETRB gene resulting in toxic megacolon (32). Transgenic expression of the ETRB gene under control of a gut-specific promoter (dopamine $\beta$-hydroxylase) rescues these rats, and they develop severe hypertension on a high-Na diet (33). Normal BP is restored by amiloride, suggesting that ETRB is involved with regulation of the epithelial $\mathrm{Na}$ channel $(\mathrm{ENaC})$ in CD cells (33). This same group (33), however, using cross-transplantation studies, concluded that salt-sensitive hypertension in rescued ETRB-deficient rats is not due to mechanisms intrinsic to the kidney (34). In these studies, transplantation of an ETRBdeficient kidney into bilaterally nephrectomized WT rats did not cause salt-sensitive hypertension. Furthermore, transplantation 


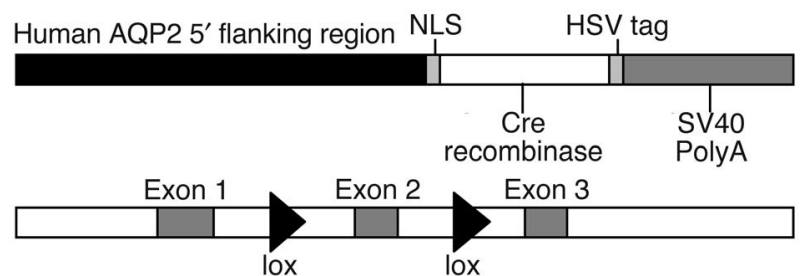

Figure 1

Constructs used in generating $\mathrm{CD}$-specific $\mathrm{KO}$ of the $E T-1$ gene. The top construct, AQP2-Cre, contains $14 \mathrm{~kb}$ of the human AQP2 promoter, an amino-terminus nuclear localization sequence (NLS), a carboxy-terminus HSV glycoprotein D epitope tag, Cre recombinase, and an SV40 PolyA sequence. The bottom construct, floxed ET-1 gene, contains loxP sites flanking exon 2 of the ET-1 gene.

of a WT kidney into bilaterally nephrectomized ETRB-deficient rats did not prevent salt-induced hypertension. Consequently, the antihypertensive effect of amiloride in ETRB-deficient rats could not be ascribed to correction of a primary defect in renal sodium excretion. These findings underscore an important consideration: Even in the rescued ETRB-deficient rats, ETRB was absent from all renal cells that normally express this receptor. Cells that normally express ETRB include most renal tubular, mesangial, glomerular epithelial, vascular smooth muscle, endothelial, and possibly interstitial cells (2). Furthermore, circulating ET-1 levels are markedly elevated in ETRB KO mice (35). Hence, it is virtually impossible to make meaningful conclusions about the physiologic or pathophysiologic role of ET-1 or ET receptors in the CD using these types of studies.

The current study describes a novel approach to examining the physiologic role of CD-derived ET-1. Mice expressing Cre recombinase under control of the aquaporin2 (AQP2) promoter were bred with mice with loxP-flanked exon 2 of the ET-1 gene to achieve CD-specific KO of ET-1, called CD ET-1 KO. We report that these mice are hypertensive and have reduced sodium excretion in response to sodium loading.

\section{Results}

Generation of mice with CD-specific KO of the ET-1 gene. AQP2-Cre mice were originally generated on a C57B16/CBA background, and the line was maintained by crossing with C57B16/CBA F1 mice. Floxed ET-1 mice were originally generated from SVJ129 ES cells; however, they were mated for over ten generations with C57Bl6/CBA F1 mice. Mice homozygous for floxed ET-1 and heterozygous for $\mathrm{AQP} 2$-Cre were generated by maintaining the $\mathrm{AQP} 2-$-Cre transgene in the maternal line. AQP2-Cre transgenic mice express Cre recombinase in postmeiotic sperm (36); when male AQP2-Cre mice are mated with ROSA26-lacZ reporter animals, ubiquitous loxP recombination occurs due to presumed Cre recombinase activity in the oocyte. In contrast, offspring from female AQP2-Cre mice show $\mathrm{CD}$-specific and male reproductive tract-specific Cre-mediated recombination $(36,37)$. CD ET-1 KO mice generated in this latter way grew and developed normally until at least 1 year of age. These animals had no gross morphologic abnormalities. In situ hybridization yielded a strong signal for ET-1 mRNA in a CD pattern primarily in renal inner medulla of the parents (floxed ET-1 and AQP2-Cre), while ET-1 mRNA was undetectable in the CDs of CD ET-1 KO mice (Figure 2). ET-1 mRNA signal intensity was similar in the parents
(Figure 2). In situ hybridization of renal cortex revealed no ET-1 mRNA in any mice (floxed ET-1, AQP2-Cre, or CD ET-1 KO). The lack of detectable renal cortical ET-1 is in agreement with other studies using in situ hybridization, immunostaining, or RT-PCR of immunodissected $\operatorname{CCD}(6,8,38,39)$. To confirm that recombination had occurred in CCDs, the ducts were microdissected and DNA PCR amplified using the primers used for genotyping. Only recombined product was seen in CCDs from CD ET-1 KO mice, while only the WT allele was seen in homozygous floxed ET-1 mice. To further demonstrate that, within the kidney, Cre was active only in the CD, AQP2-Cre mice were bred with ROSA26YFP reporter mice, and kidney sections were immunostained for AQP2. As shown in Figure 3, AQP2 expression and YFP fluorescence precisely overlap within inner medulla, outer medulla, and cortex. In situ hybridization for the ET-1 message was also performed on lung and stomach from the parental strains and in CD ET-1 KO mice. No difference in ET-1 mRNA signal intensity was observed between all three lines, supporting the tissue specificity of CD ET-1 KO. Finally, and as predicted, plasma ET-1 levels were not affected in ET-1 mice as compared with mice containing the unrecombined ET-1 gene (Table 3). Urinary ET-1 was reduced in CD ET-1 KO mice as compared with unrecombined floxed ET-1 animals (Table 3). In summary, CD ET-1 KO mice have $\mathrm{CD}$-specific inactivation of the ET-1 gene. Although not specifically tested, it was presumed that ET-1 was also disrupted in male reproductive tract.

Normal Na diet. Systolic BP and pulse rate were determined in floxed CD ET-1 KO and littermates that were homozygous for floxed ET-1 but contained no Cre recombinase (hereafter referred to as controls). CD ET-1 KO mice were hypertensive as compared with controls (Table 1 and Figures 4 and 5). Pulse rate was not significantly different between the groups (Table 1). There was no significant difference in BP or pulse rate between males and females in CD ET-1 KO mice, indicating that ET-1 gene disruption in the male reproductive tract did not influence this phenotype.
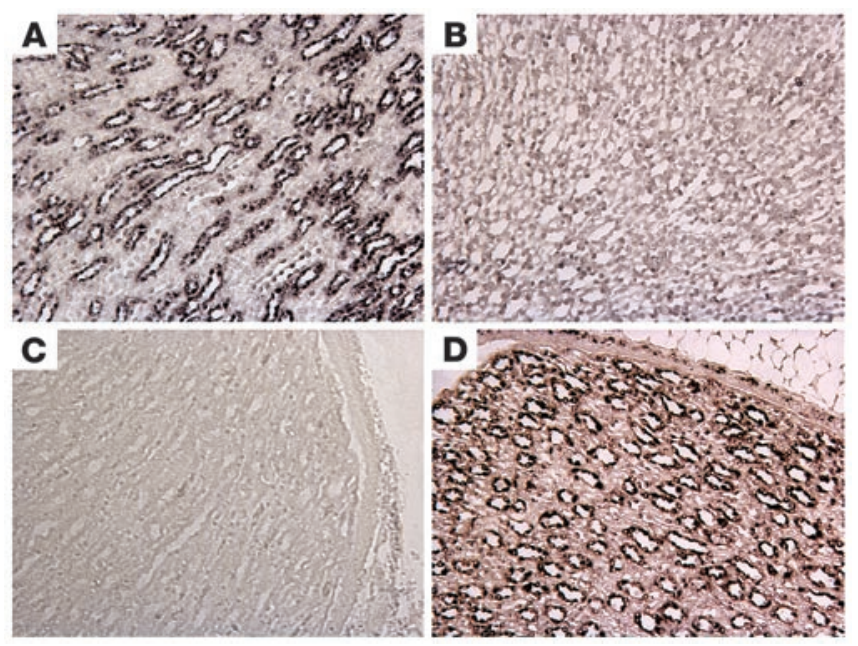

\section{Figure 2}

In situ hybridization using an antisense probe for ET-1 mRNA in renal inner medulla from mice containing floxed ET-1 (A), CD ET-1 KO (B), and only the AQP2-Cre transgene (D). (C) The results obtained using the sense probe for $E T-1$ mRNA in floxed mice. Note that the staining is in a CD pattern. Images shown $(\times 400)$ are representative of five mice in each group. 


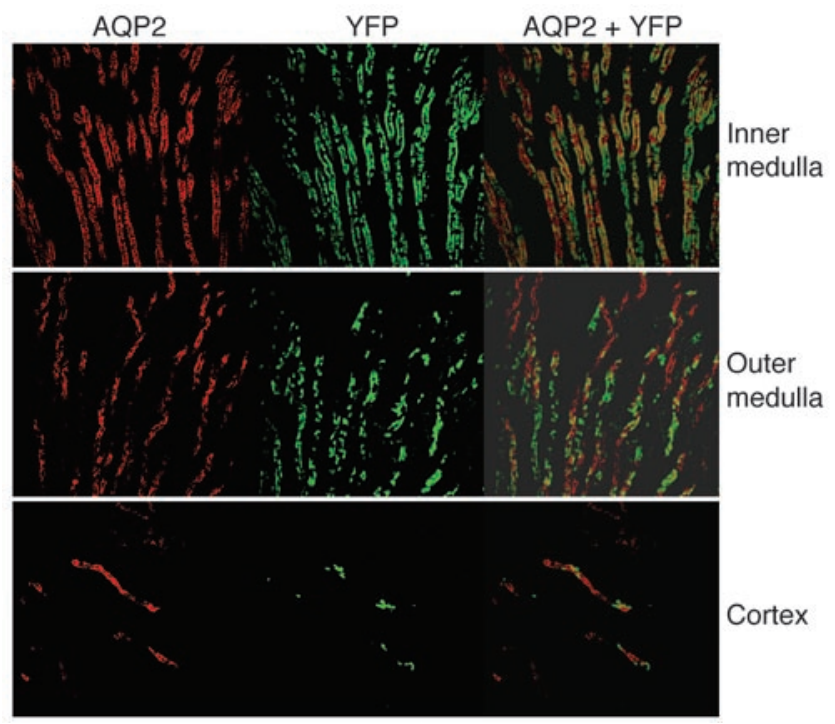

Figure 3

Colocalization of YFP expression and AQP2 immunofluorescence in mice doubly heterozygous for ROSA26-YFP and AQP2-Cre. A representative photomicrograph is shown from five separate animals. Images are $\times 600$.

Renal function was assessed to determine if hypertension in CD ET-1 KO mice on a normal Na diet was associated with altered salt and/or water excretion. Importantly, all mice had precisely matched food and water intake. This was achieved by ration feeding them a gelled diet that met all their nutritional needs and provided a normal amount of water. Mice liked the gel and ate everything provided $(9 \mathrm{ml} /$ day). Because of the gel's consistency, mice did not break off any pieces and drop them into the bottom of the cage. No water bottles were used - all the water came from the gels. This prevented the large error normally found when measuring water intake in mice on a normal diet. Thus, we were certain that any differences in urinary excretion were not due to changes in intake.

Despite the hypertension in CD ET-1 KO mice, there were no differences in body weight, urine volume, urine $\mathrm{Na}$ or K excretion, urine or plasma osmolality, plasma aldosterone concentration, or plasma renin activity (PRA) (Tables 1 and 3). Creatinine clearance was similar between floxed ET-1 and CD ET-1 KO mice, suggesting that within the limits of these measurements glomerular filtration rate was unchanged by $\mathrm{CD}$ ET-1 KO. As expected, plasma ET-1 concentration was unaffected by CD KO of ET-1 (Table 3). Urine volume is substantially less than water intake; this is typical of mice (they have high insensible fluid loss). These data indicate that hypertension occurs in CD ET-1 KO mice on a normal Na diet, but it is not due to ongoing renal $\mathrm{Na}$ and water retention or changes in aldosterone levels or PRA.

High-Na diet. Essential hypertension may result from initial $\mathrm{Na}$ retention, followed by resetting of the pressure-natriuresis curve and return to Na balance. Thus, CD ET-1 KO mice were Na loaded to attempt to uncover an impaired natriuretic response. Mice were fed a high-Na diet (eightfold increase in $\mathrm{Na}$ ) for up to 2 weeks. Systolic BP rose in CD ET-1 KO mice but did not change in controls (Figure 4 and Table 2). This rise occurred on the first day of the high-Na diet and remained elevated thereafter for the duration of the experiment (Figure 4). Coincident with this, CD ET-1 KO mice had reduced $\mathrm{Na}$ excretion that was evident over the first 3 days of the high-Na diet (Figure 4 and Table 2). After the first 3 days, urinary $\mathrm{Na}$ excretion was similar between the two groups and indicated return to $\mathrm{Na}$ balance. Table 2 shows the urine, blood, hemodynamic, and weight parameters on day 2 of a high-Na diet, the day when $\mathrm{Na}$ retention was maximal in CD ET-1 KO mice. Urine volume tended to be less, but this did not achieve statistical significance. Na loading caused a comparable fall in plasma aldosterone and PRA in CD ET-1 KO mice and controls. ET-1 excretion rose in both groups, but to a lesser degree in CD ET-1 KO mice. There was no difference in $\mathrm{ClCr}$ or $\mathrm{K}$ excretion between the groups. Thus, CD ET-1 KO mice have reduced ability to excrete a Na load associated with weight gain and worsened hypertension.

Diuretic studies. To determine if CD-derived ET-1 regulates CD $\mathrm{Na}$ reabsorption through modulation of $\mathrm{ENaC}$ activity, amiloride ( $3 \mathrm{mg} / \mathrm{kg}$ per body weight per day intraperitoneally) was administered to CD ET-1 KO and control mice on normal or high-Na diets (amiloride started simultaneously with normal or high-Na diet). Amiloride inhibited Na-loading exacerbation of hypertension in CD ET-1 KO mice on a high-Na diet and slightly reduced systolic BP in CD ET-1 KO mice on a normal Na diet (Figure 5). Amiloride did not significantly change systolic BP in control mice on normal or high-Na diets (Figure 5). Amiloride did prevent $\mathrm{Na}$ loading-induced excessive Na retention in CD ET-1 KO mice (Figure 6). Thus, baseline and salt-sensitive hypertension in CD ET-1 $\mathrm{KO}$ mice is partially due to amiloride-inhibitable pathways.

To determine if another natriuretic agent would also ameliorate hypertension in CD ET-1 KO mice, furosemide $(4.2 \mathrm{mg} / \mathrm{kg} /$ day) was administered to CD ET-1 KO and control mice on normal or high-Na diets (furosemide started simultaneously with normal or high-Na diet). Furosemide ameliorated Na-loading exacerbation of hypertension and excessive Na retention in CD ET-1 KO mice on a high-Na diet (Figures 5 and 6). Furosemide did not reduce systolic BP in CD ET-1 KO mice on a normal Na diet (Figure 5). Furosemide, like amiloride, did not significantly change systolic $\mathrm{BP}$ in control mice on normal or high-Na diets (Figure 5).

\section{Table 1}

Metabolic balance data in control and CD ET-1 KO mice on a normal $(0.3 \%)$ sodium diet

\begin{tabular}{lcc}
\hline & Control & CD ET-1 KO \\
Systolic BP (mmHg) & $93 \pm 6$ & $114 \pm 5^{\mathrm{A}}$ \\
Pulse (bpm) & $587 \pm 45$ & $603 \pm 38$ \\
Weight $(\mathrm{g})$ & $22.3 \pm 1.5$ & $23.1 \pm 1.8$ \\
Gel intake (g) & $9 \pm 0.1$ & $8.9 \pm 0.1$ \\
Urine volume (ml/day) & $2.4 \pm 0.5$ & $2.4 \pm 0.6$ \\
Urine [Na] (mEq/l) & $345 \pm 45$ & $357 \pm 37$ \\
Urine [K] (mEq/l) & $132 \pm 19$ & $135 \pm 24$ \\
Urine 0sm (m0sm/l) & $2,613 \pm 351$ & $2,685 \pm 402$ \\
Urine [creatinine] (mg/dl) & $22.1 \pm 0.9$ & $19.2 \pm 3.0$ \\
Plasma [Na] (mEq/l) & $142 \pm 2$ & $140 \pm 3$ \\
Plasma [K] (mEq/l) & $4.6 \pm 0.2$ & $4.6 \pm 0.3$ \\
Plasma osm (m0sm/l) & $309 \pm 3$ & $313 \pm 3$ \\
Plasma [creatinine] (mg/dl) & $0.28 \pm 0.04$ & $0.27 \pm 0.02$ \\
Urine Na excretion (mEq/day) & $0.90 \pm 0.13$ & $0.84 \pm 0.15$ \\
Urine K excretion (mEq/day) & $0.34 \pm 0.05$ & $0.32 \pm 0.06$ \\
CICr (ml/min) & $0.13 \pm 0.01$ & $0.12 \pm 0.04$ \\
& & \\
\hline
\end{tabular}

$n=9-18$ per data point. ${ }^{A} P<0.01$ vs. control. bpm, beats per minute; osm, osmolality. 


\section{Table 2}

Metabolic balance data in control and CD ET-1 KO mice on day 2 of a high-sodium diet

\begin{tabular}{lcc}
\hline & Control & CD ET-1 KO \\
Systolic BP (mmHg) & $95 \pm 8$ & $130 \pm 7$ A \\
Pulse (bpm) & $611 \pm 20$ & $598 \pm 32$ \\
Weight $(\mathrm{g})$ & $23.8 \pm 2.8$ & $26.1 \pm 2.4$ \\
Weight change vs. previous day (g) & $+0.7 \pm 0.3$ & $+1.5 \pm 0.4 \mathrm{~B}$ \\
Total intake (g) & $17.8 \pm 0.2$ & $17.7 \pm 0.2$ \\
Urine volume (ml/day) & $11.6 \pm 2.1$ & $9.6 \pm 3.2$ \\
Urine [Na] (mEq/l) & $521 \pm 61$ & $509 \pm 48$ \\
Urine [K] (mEq/l) & $38 \pm 5$ & $41 \pm 6$ \\
Urine 0sm (m0sm/l) & $1,693 \pm 321$ & $1,662 \pm 294$ \\
Urine [creatinine] (mg/dl) & $4.9 \pm 0.8$ & $6.1 \pm 0.9$ \\
Plasma [Na] (mEq/l) & $139 \pm 2$ & $141 \pm 3$ \\
Plasma [K] (mEq/l) & $4.5 \pm 0.2$ & $4.5 \pm 0.3$ \\
Plasma 0sm (m0sm/l) & $307 \pm 5$ & $304 \pm 4$ \\
Plasma [creatinine] (mg/dl) & $0.27 \pm 0.03$ & $0.28 \pm 0.03$ \\
Urine Na excretion (mEq/day) & $6.01 \pm 0.48$ & $4.93 \pm 0.59 \mathrm{C}$ \\
Urine K excretion (mEq/day) & $0.42 \pm 0.08$ & $0.38 \pm 0.07$ \\
ClCr (ml/min) & $0.14 \pm 0.03$ & $0.14 \pm 0.02$ \\
& &
\end{tabular}

$n=9$ per data point. ${ }^{A} P<0.001$ vs. control; ${ }^{B} P<0.025$ vs. control;

$\mathrm{C} P<0.01$ vs. control.

\section{Discussion}

The current studies demonstrate that CD-derived ET-1 is an important physiologic regulator of systemic BP and renal $\mathrm{Na}$ and water excretion. Deficiency of CD-derived ET-1 leads to baseline (normal Na diet) hypertension as well as salt-sensitive hypertension. CD ET-1 KO mice also have impaired ability to excrete a $\mathrm{Na}$ load. These studies are also the first to describe hypertension resulting from deficiency or mutation of a single nontransporter protein in a specific nephron segment. Importantly, these studies employed the AQP2 promoter to drive Cre recombinase expression - AQP2 is normally expressed throughout the entire CD as well as in the connecting segment. In contrast, the HoxB7 promoter has recently been used to drive Cre expression specifically in CDs; however, this promoter has the disadvantage of not being active in the connecting segment and perhaps not in early CCDs (40).

The role of ET-1 in hypertension has been intensively investigated (41); however, relatively little attention has been paid to the involvement of intrarenal ET-1 in the pathogenesis and/or maintenance of hypertension. Medullary ET-1 production is reduced in some animal models of hypertension, including SHR, Dahl S, and Prague hypertensive rats, suggesting that decreased ET-1-mediated inhibition of CD Na and water reabsorption contributes to the hypertensive state (42-45). These findings are supported by the observation that acutely isolated or cultured IMCD cells from hypertensive SHR rats synthesize less ET-1 as compared with IMCD cells from normotensive WKY controls (43). Several groups observed that humans with essential hypertension, and particularly those with salt-sensitive hypertension, excrete less urinary ET-1 than do normotensive subjects (46-48), although one group reported increased urinary ET-1 in salt-sensitive as compared with salt-resistant hypertensive patients (49). In sum- mary, these studies suggest that renal medullary and, in particular, CD ET-1 production may be reduced in the setting of genetic hypertension. Taken together with the finding that deficiency of CD-derived ET-1 leads to salt retention and hypertension, these studies provide an impetus to more closely examine renal ET-1 production in patients with essential hypertension.

The mechanisms by which deficiency of CD-derived ET- 1 causes renal $\mathrm{Na}$ and water retention and hypertension remain to be fully determined. In vitro studies suggest that ET-1 may act in an autocrine manner by activation of CD ETRBs to inhibit AVP-stimulated cAMP accumulation (13-15), decrease Na/K-ATPase activity (20), and inhibit ENaC activity (50). Both amiloride (at a dose specific for $\mathrm{ENaC}$; see refs. 51, 52) and furosemide ameliorated salt-sensitive hypertension in CD ET-1 KO mice, supporting the notion that renal $\mathrm{Na}$ retention is pathogenic in the hypertensive response to $\mathrm{Na}$ loading. Nonetheless, it is not possible to infer that the $\mathrm{CD}$, or the thick ascending limb, are the principal sites of $\mathrm{Na}$ retention in this model simply because these are the sites of action of the diuretics used. CD-derived ET-1 may also influence $\mathrm{Na}$ and water reabsorption through paracrine actions. Renal medullary interstitial cells are a likely target of CD-derived (and particularly IMCD-derived) ET-1. Interstitial cells are present in abundance in the inner medulla and are adjacent to the CD. These cells are a likely target of ET-1; they express ET receptors (53), but do not synthesize the peptide (54). ET-1 induces cultured medullary interstitial cell nitric oxide and $\mathrm{PGE}_{2}$ production $(55,56)$. It is conceivable that such nitric oxide and/or $\mathrm{PGE}_{2}$ released in response to ET-1 could inhibit $\mathrm{Na}$ reabsorption by the $\mathrm{CD}$. In addition, these agents may modulate medullary blood flow (MBF). Exogenous ET-1 constricts isolated outer medullary descending vasa recta (57), suggesting that the peptide reduces MBF. In contrast, in vivo studies show that ET-1 increases MBF. Two groups found that intravenous ET-1 markedly decreased rat renal cortical blood flow associated with an increase in $\operatorname{MBF}(58,59)$. It is possible, therefore, that CD-derived ET-1 may indirectly affect MBF through stimulation of vasodilator release from medullary interstitial cells.

Finally, the possibility must be considered that CD-derived ET-1 modulates endocrine factors. Although not fully characterized, medullipins are cytochrome P450 lipid metabolites produced by medullary interstitial cells that cause hypotension, diuresis, and natriuresis (60). Since ET-1 has been described to increase renal cytochrome P450 activity (61), it is tempting to speculate that CDderived ET-1 might modulate medullary interstitial cell medullipin release. We also observed that, despite the different $\mathrm{BP}$ responses to Na loading, CD ET-1 KO and control mice had similar levels of and suppression of plasma aldosterone and PRA. Thus, for the degree
Table 3

Plasma and urine hormone levels in control and ET-1 KO mice under varying dietary conditions

\begin{tabular}{lccccc}
\hline & & $\begin{array}{c}\text { Urine ET-1 } \\
(\mathbf{p g} / \mathbf{d a y})\end{array}$ & $\begin{array}{c}\text { Plasma ET-1 } \\
\mathbf{( p g / m l )}\end{array}$ & $\begin{array}{c}\text { Aldosterone } \\
\mathbf{( p g / d a y )}\end{array}$ & $\begin{array}{c}\text { PRA } \\
(\mathbf{p g A l} / \mathbf{\mu l} / \mathbf{h})\end{array}$ \\
Normal Na & Control & $16.2 \pm 2.8$ & $5.3 \pm 0.8$ & $0.93 \pm 0.05$ & $0.42 \pm 0.18$ \\
& CD ET-1 KO & $10.1 \pm 2.4^{\mathrm{A}}$ & $5.6 \pm 0.9$ & $1.01 \pm 0.06$ & $0.50 \pm 0.22$ \\
High Na & Control & $31.2 \pm 4.1$ & $5.0 \pm 1.2$ & $0.50 \pm 0.03$ & $0.32 \pm 0.11$ \\
& CD ET-1 KO & $16.8 \pm 3.6^{\mathrm{B}}$ & $5.1 \pm 1.3$ & $0.50 \pm 0.03$ & $0.36 \pm 0.10$ \\
\hline
\end{tabular}

$n=5-9$ per data point. ${ }^{\mathrm{A} P}<0.01$ vs. control, ${ }^{\mathrm{B} P}<0.001$ vs. control. Aldosterone, urinary aldosterone excretion; pgAl, pg angiotensin I. 

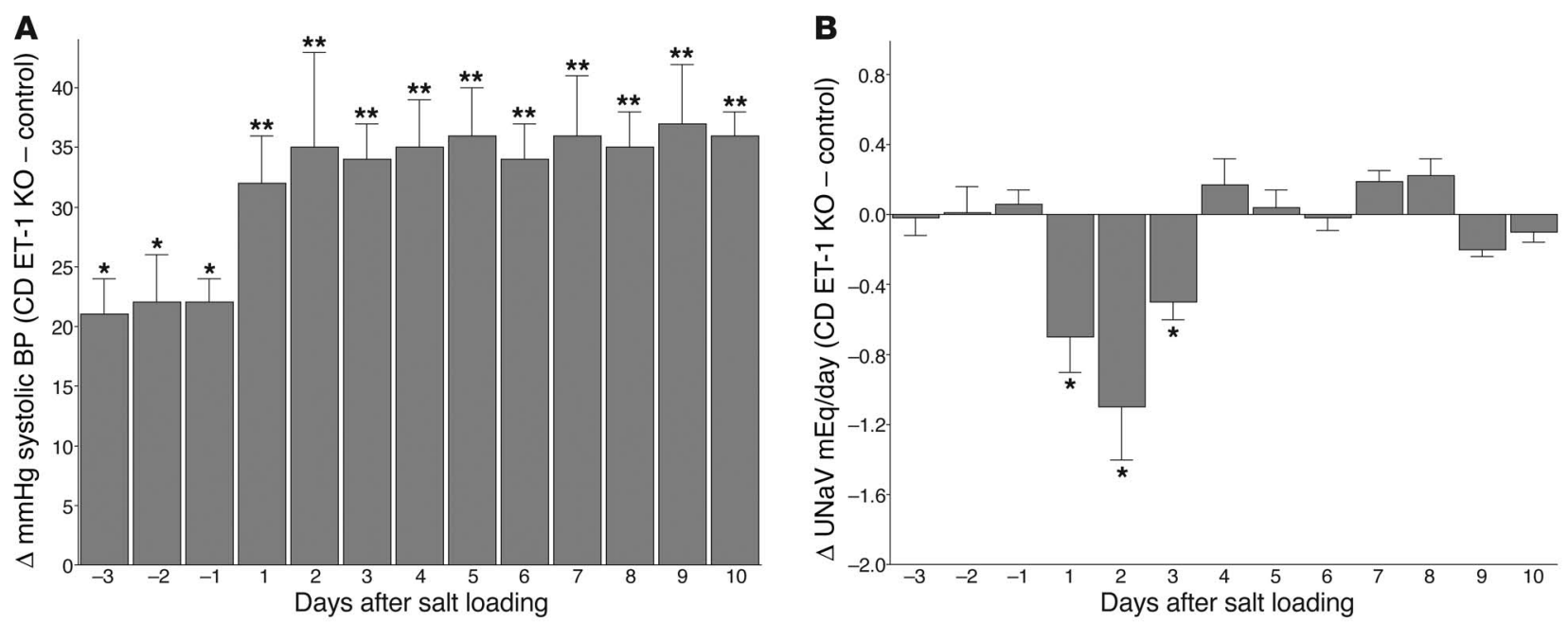

Figure 4

Differences in systolic BP $(\mathbf{A})$ and urinary sodium excretion $(\mathrm{UNaV})(\mathrm{B})$ in control and CD ET-1 KO mice on a normal or high-Na diet. $n=9$ per data point. ${ }^{*} P<0.01$ vs. control; ${ }^{* \star} P<0.001$ vs. control and $P<0.01$ vs. CD ET-1 KO normal Na diet.

of hypertension, the renin-aldosterone axis was not apparently appropriately suppressed in CD ET-1 KO mice. The reasons for this observation remain speculative, including relative insensitivity of the renin-angiotensin-aldosterone system in rodents, release of factors from kidneys of CD ET-1 KO mice that modulate this system, or relatively poor sensitivity of the assays employed. While not necessarily analogous to $\mathrm{CD}$-specific $\mathrm{KOs}$, it is nonetheless interesting to note that PRA and plasma aldosterone levels were also not different between salt-loaded normotensive control rats and severely hypertensive rats homozygous for a naturally occurring mutation in the ETRB (33). In summary, CD-derived ET-1 may act through a variety of autocrine, paracrine, or endocrine mechanisms to reduce $\mathrm{BP}$ and induce a natriuresis and diuresis.

The findings in the current study beg the question as to why a CD-derived factor would be an important physiologic regulator of systemic $\mathrm{BP}$ and renal $\mathrm{Na}$ and water excretion. It is notable that the bulk of CD-derived ET-1 is made in the medullary CD, the site of final regulation of urine sodium and water content. Factors that regulate CD ET-1 production should, therefore, be able to substantially impact urinary $\mathrm{Na}$ and water excretion. Renal medullary ET-1 production is markedly enhanced by volume expansion, while dehydration reduces renal medullary ET-1 content (62). The factors mediating volume-related changes in medullary ET-1 production are unknown. Identification of such factors is important in understanding how the kidney can sense and appropriately respond to changes in extracellular fluid volume status.

\section{Methods}

Generation of transgenic mice. Three types of mice were used: loxP-flanked (floxed) ET-1 gene (SVJ129 × C57BL6/CBA); AQP2-Cre (C57BL6/CBA); and AQP2-Cre $\times$ floxed ET-1 (Figure 1). Floxed ET-1 mice were obtained from Masashi Yanagisawa at the Howard Hughes Institute at University of Texas Southwestern Medical Center. These mice contain loxP sites inserted into introns 1 and 2 in the ET-1 gene (flanking the critical exon 2 that was targeted in the original traditional ET-1 KO mice; ref. 30) by homologous recombination in ES cells. The sequence for this mutation has been confirmed in M. Yanagisawa's laboratory and our laboratory. AQP2-Cre mice contain a transgene with $14 \mathrm{~kb}$ of the human $A Q P 2$ gene
5' flanking region driving expression of Cre recombinase (36). An SV40 nuclear localization signal is located on the amino terminus of Cre, and an 11-amino acid epitope tag, derived from herpes simplex virus (HSV) glycoprotein D, is located on the carboxy terminus of Cre. The polyadenylation signal from the SV40 late region, without an intron, was also added for efficient expression. Female AQP2-Cre mice were mated with male floxed ET-1 mice; female offspring heterozygous for both AQP2-Cre and floxed ET-1 were bred with males homozygous for floxed ET-1. Animals homozygous for floxed ET-1 and heterozygous for AQP2-Cre (CD ET-1 KO) were used in all studies.

Genotyping. Tail DNA was prepared by standard methods and PCR amplified for the AQP2-Cre transgene using oligonucleotide primers hAQP2 F2 (5'-AGTCAGAGAGATGGGGGCCGG-3') and CreTag R

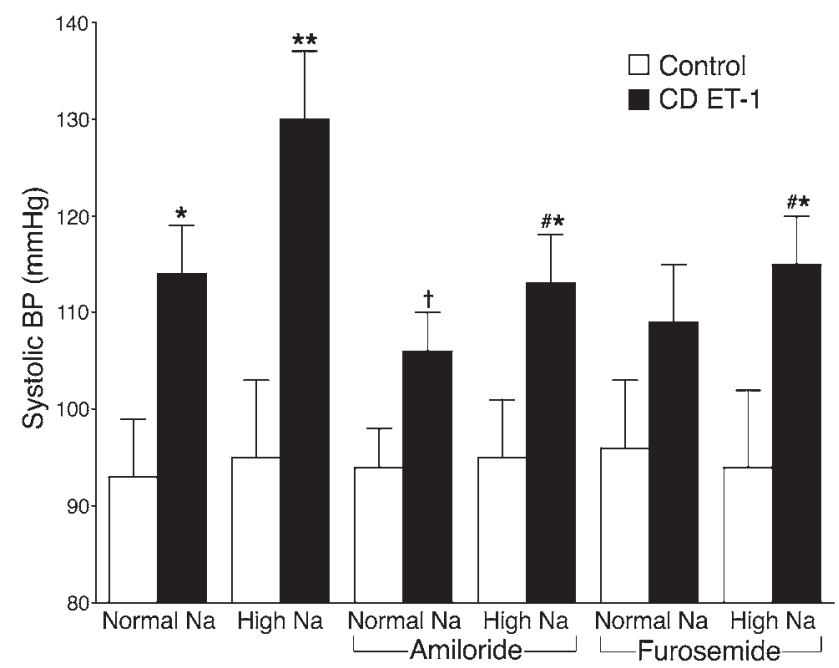

Figure 5

Systolic BP in control and CD ET-1 KO mice on a normal or high-Na diet with or without amiloride (3 $\mathrm{mg} / \mathrm{kg} / \mathrm{day})$ or furosemide $(4.2 \mathrm{mg} /$ $\mathrm{kg} /$ day). $n=5-9$ per data point. ${ }^{*} P<0.01$ vs. control, ${ }^{*} P<0.001$ vs. control and $P<0.01$ vs. CD ET- $1 \mathrm{KO}$ normal Na; ${ }^{\dagger} P<0.05$ vs. ET-1 normal $\mathrm{Na} ;{ }^{\#} P<0.01$ vs. CD ET-1 KO high $\mathrm{Na}$. 


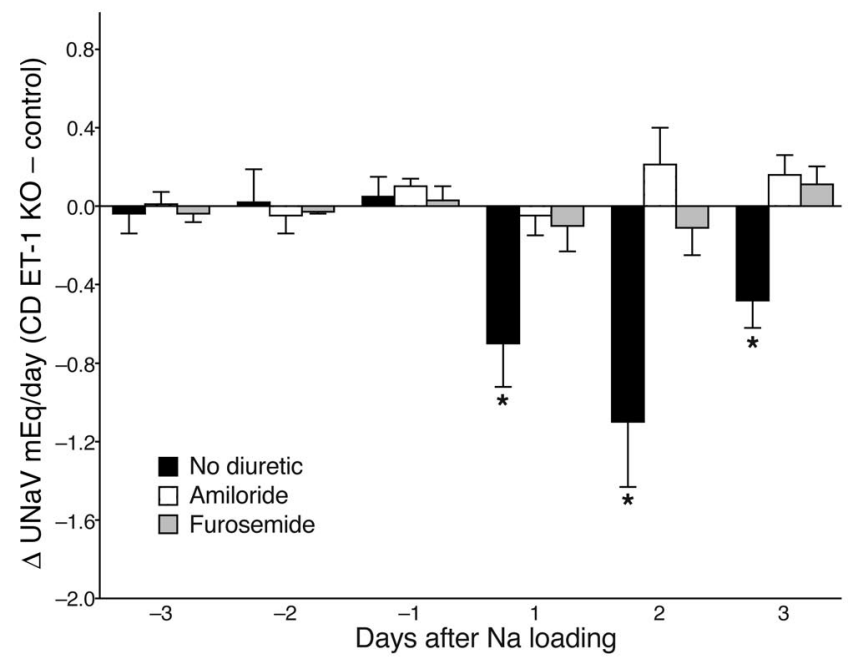

Figure 6

Differences in urinary sodium excretion between control and $C D$ ET-1 KO mice on a normal or high-Na diet. $n=6-9$ per data point. ${ }^{\star} P<0.01$ vs. control.

(5'-GCGAACATCTTCAGGTTCTGCGG-3'), which amplify the 730-bp junction between the AQP2 promoter and the Cre gene. Genotyping the ET-1 gene involved three primer sets. The first set used primers ET-1CF (5'-GCTGCCCAAAGATTCTGAATTC-3') and ET-1BR (5'-GATGATGTCCAGGTGGCAGAAG-3'), which amplify 800 bp of the endogenous ET-1 allele. The second set used primers ET-1AF (5'-CCCAAAGATTCTGAATTGATAACTTCG-3') and ET-1BR (5'-GATGATGTCCAGGTGGCAGAAG$3^{\prime}$ ), which amplify the same 800-bp region; however, the forward primer overlaps loxP and hence recognizes only the floxed ET-1 allele. The third set used primers ET-1AF and ET-1DR (5'-AACCTCCCAGTCCATACGGTAC- $3^{\prime}$ ), which amplify the region in the targeted allele spanning the loxP sites. The PCR product of the nonrecombined allele is $2 \mathrm{~kb}$, while the recombined allele yields a $300-b p$ product. PCR products were visualized after electrophoresis through $1.5 \%$ agarose.

In situ bybridization. Organs were fixed in $10 \%$ buffered formalin, embedded in paraffin, and 5 - to $10-\mu \mathrm{m}$ sections were made. Sections were rehydrated and post-fixed in $4 \%$ paraformaldehyde. Some sections were stained with $\mathrm{H} \& \mathrm{E}$, while in most, endogenous peroxidase activity was quenched with $1.5 \% \mathrm{H}_{2} \mathrm{O}_{2}$ and sections acetylated with $0.1 \mathrm{M}$ triethanolamine ( $\mathrm{pH} 8.0$ ) containing $0.25 \%$ acetic anhydride. Sections were prehybridized for 2 hours and then hybridized overnight at $42^{\circ} \mathrm{C}$ with $125 \mathrm{ng} / \mathrm{ml}$ digitonin-labeled (DIG-labeled) riboprobes complementary to antisense and sense mouse ET-1 cDNA. The prehybridization and hybridization buffer contained $50 \%$ deionized formamide, $10 \%$ dextran sulfate, $4 \times$ SSC, $250 \mu \mathrm{g} / \mathrm{ml}$ transfer RNA (tRNA), $250 \mu \mathrm{g} / \mathrm{ml}$ single stranded DNA (ssDNA), and $10 \mathrm{mM}$ DTT. Slides were washed, incubated in 1:10 dilution of blocking reagent in maleic acid buffer (Roche Diagnostics, Indianapolis, Indiana, USA), and incubated with 1:100 dilution of anti-DIG-POD $\mathrm{Ab}$ in blocking solution (Roche Diagnostics) for 1-2 hours. Sections were then washed, developed for a color reaction with DAB (SK-4100; Vector Laboratories, Burlingame, California, USA), rinsed, and viewed.

For riboprobes, pmET1, containing $380 \mathrm{bp}$ of the SacI-HindIII fragment of mouse ET-I cDNA in pBluescript II, was used. This fragment is complementary to ET-1 mRNA that is encoded by all of exon 2 and part of exon 3. The 380-bp ET-1 cDNA insert was excised, blunted with T4 DNA polymerase, phenol/chloroform extracted, and used for generation of DIG riboprobes with the DIG RNA labeling mix (Roche Diagnostics), along with standard in vitro transcription components $(0.5 \mu \mathrm{g}$ linearized template, $10 \mathrm{mM}$ DTT, $1 \mathrm{U} / \mu \mathrm{l}$ RNAsin, $1 \mathrm{U} / \mu \mathrm{l}$ RA polymerase). The transcription reaction was carried out for 2 hours, DNase I was added, and the probe ethanol was precipitated.

Colocalization of Cre activity and AQP2 expression. AQP2-Cre mice were bred with ROSA26-YFP mice (generous gift of Frank Costantini, Columbia University, New York, New York, USA; ref. 63), and doubly heterozygous offspring were analyzed. Cells expressing active Cre recombinase excise the floxed STOP sequence downstream of the ROSA26 promoter with resultant YFP expression. Kidneys were sectioned $(200 \mu \mathrm{m})$ on a vibratome (OTS-3000; Electron Microscopy Sciences, Fort Washington, Pennsylvania, USA), fixed and immunostained for AQP2 using an affinity-purified rabbit polyclonal anti-AQP2 Ab (generous gift of Mark Knepper, NIH, Bethesda, Maryland, USA) as previously described (36). Sections were then incubated with Cy5-conjugated anti-rabbit IgG (Vector Laboratories) and visualized by confocal microscopy.

Metabolic balance studies. All mice used were 2-4 months old. All mice were acclimated for 1 week to Nalgene Labware metabolic cages (Rochester, New York, USA). Mice were fed $9 \mathrm{ml}$ of a gelled diet that contained all nutrients and water. The gelled food was made by dissolving $65.7 \mathrm{~g}$ of PMI rodent powdered diet (LD101; LabDiet, Richmond, Indiana, USA) and 7 g gelatin in $110 \mathrm{ml}$ water. $\mathrm{NaCl}$ was added to give a final sodium concentration of $0.3 \%$. The gel was solidified in plastic scintillation vials and served as the sole source of food and water. BP and pulse were determined daily by tailcuff plethysmography (BP-2000; Visitech Systems, Apex, North Carolina, USA); the values were not recorded during this conditioning period. After 1 week, metabolic balance studies were performed for 3 consecutive days. Daily gel intake was measured and urine collected under oil. BP and pulse were determined daily and were averaged from 8-10 recordings each day. Mice were sacrificed at this point by exsanguination by cardiac puncture under halothane and Avertin anesthesia.

For sodium-loading studies, mice were studied as described above except that after 3 days of a normal $(0.3 \%)$ sodium diet, they were placed on a high-sodium diet for 10 days. The high-sodium diet consisted of $9 \mathrm{ml}$ of gelled diet containing $1 \%$ sodium. In addition, mice were given normal saline to drink. Daily weights and vital signs were obtained and urine collected. At the end of this period, mice were exsanguinated.

For amiloride studies, mice were studied as described above except that they were given $3 \mathrm{mg} / \mathrm{kg}$ body weight amiloride in $100 \mu \mathrm{l}$ normal saline intraperitoneally daily during either the normal or the high-Na diets. Daily weights and vital signs were obtained and urine collected. At the end of this period, mice were exsanguinated. For furosemide studies, mice were similarly treated except that osmotic minipumps (1003D; Alzet Corp., Cupertino, California, USA) containing furosemide $(12.5 \mathrm{mg} / \mathrm{kg}$ delivered over 3 days at $1 \mu \mathrm{l} / \mathrm{h}$ ) were implanted subcutaneously under ketamine $(100 \mathrm{mg} / \mathrm{kg})$ and xylazine $(5 \mathrm{mg} / \mathrm{kg})$ anesthesia immediately prior to being studied on a normal or high-Na diet.

The ethics of all animal experiments were approved by the University of Utah Institutional Animal Care and Use Committee.

Electrolyte and hormone analysis. Plasma from all animals was analyzed for $\mathrm{Na}$ and $\mathrm{K}$ concentrations (EasyVet analyzer; Medica Corp., Bedford, Massachusetts, USA), osmolality (Osmett II; Precision Systems, Natick, Massachusetts, USA), and creatinine (Jaffe colorimetry method using a commercially available kit from Sigma-Aldrich, St. Louis, Missouri, USA). Plasma from exsanguinated mice was also analyzed for ET-1 and PRA. For ET-1 determination, heparinized samples $(100 \mu \mathrm{l})$ were applied to a 100-mg Sep-Pak C18 cartridge (Waters Corp., Milford, Massachusetts, USA), equilibrated and eluted as previously described (64), and ET-1 determined by radioimmunoassay (Peninsula Laboratories Inc., San Carlos, California, USA). PRA was measured as the amount of Ang I generated 
after incubation with excess angiotensinogen as previously described (65). Two microliters of plasma were incubated for 20 minutes at $37^{\circ} \mathrm{C}$ with excess porcine angiotensinogen $(4 \mu \mathrm{M}$; Sigma-Aldrich) in a $10-\mu \mathrm{l}$ reaction containing sodium acetate $(50 \mathrm{mM}, \mathrm{pH} 6.5)$, aminoethylbenzene sulfonyl fluoride (2.5 mM), 8-hydroxyquinoline (1 mM), and EDTA (5 mM). The reaction was stopped by boiling, and Ang I measured using an indirect radioimmunoassay (NEN-DuPont, Boston, Massachusetts, USA). Results are expressed as Ang I generated $/ \mathrm{h} / \mu \mathrm{l}$ plasma.

Urine was measured for ET-1, volume, $\mathrm{Na}, \mathrm{K}$, and osmolality as described above. Urine from basal and sodium-loading studies was analyzed for 24hour aldosterone excretion. Urine was hydrolyzed with $\mathrm{HCl}$, ethyl acetate was extracted, and aldosterone was determined by radioimmunoassay (Coat-a-Count; Diagnostic Products Inc., Los Angeles, California, USA).

Statistics. Comparisons between floxed ET-1 and CD ET-1 KO mice were analyzed by the unpaired Student's $t$ test. Comparisons within floxed ET-1 and CD ET-1 KO mice were analyzed by the paired Student's $t$ test. Comparisons between systolic BPs in three lines of mice as well as mice on high-sodium or normal sodium diets plus or minus amiloride or furosemide were made using ANOVA with the Bonferroni correction.
$P$ values less than 0.05 were taken as significant. Data are expressed as mean plus or minus SEM.

\section{Acknowledgments}

The technical assistance of Elaine Hillas in Jean-Marc Lalouel's laboratory is appreciated. Some of the initial electrolytes were determined by Robert Baranowski in Christof Westenfelder's laboratory. This research was funded in part by grants from the NIH (DK-59047 and DK-58953 to D.E. Kohan).

Received for publication January 14, 2004, and accepted in revised form June 29, 2004.

Address correspondence to: Donald E. Kohan, Division of Nephrology, University of Utah Health Sciences Center, 1900 East, 30 North, Salt Lake City, Utah 84132, USA. Phone: (801) 581-6709; Fax: (801) 581-4343; E-mail: donald.kohan@hsc.utah.edu.

Dowhan Ahn and Yuqiang Ge contributed equally to this work.
1. Yanagisawa, M., et al. 1988. A novel potent vasoconstrictor peptide produced by vascular endothelial cells. Nature. 332:411-415.

2. Kohan, D. 1997. Endothelins in the normal and diseased kidney. Am. J. Kidney Dis. 29:2-26.

3. Kitamura, K., Tanaka, T., Kato, J., Eto, T., and Tanaka, K. 1989. Regional distribution of immunoreactive endothelin in porcine tissue: abundance in inner medulla of kidney. Biochem. Biophys. Res. Commun. 161:348-352.

4. Kohan, D.E. 1991. Endothelin synthesis by rabbit renal tubule cells. Am. J. Physiol. 261:F221-F226.

5. Chen, M., et al. 1993. Endothelin-1 mRNA in glomerular and epithelial cells of kidney. Am. J. Physiol. 265:F542-F550.

6. Pupilli, C., et al. 1994. Presence and distribution of endothelin-1 gene expression in human kidney. Am. J. Physiol. 267:F679-F687.

7. Uchida, S., Takemoto, F., Ogata, E., and Kurokawa, K. 1992. Detection of endothelin-1 mRNA by RTPCR in isolated rat renal tubules. Biochem. Biophys. Res. Commun. 188:108-113.

8. Ujiie, K., et al. 1992. Messenger RNA expression and synthesis of endothelin-1 along rat nephron segments. J. Clin. Invest. 90:1043-1048.

9. Takemoto, F., Uchida, S., Ogata, E., and Kurokawa, K. 1993. Endothelin-1 and endothelin-3 binding to rat nephrons. Am. J. Physiol. 264:F827-F832.

10. Terada, Y., Tomita, K., Nonoguchi, H., and Marumo, F. 1992. Different localization of two types of endothelin receptor mRNA in microdissected rat nephron segments using reverse transcription and polymerase chain reaction assay. J. Clin. Invest. 90:107-112.

11. Chow, L.H., Subramanian, S., Nuovo, G.J., Miller, F., and Nord, E.P. 1995. Endothelin receptor mRNA expression in renal medulla identified by in situ PT-PCR. Am. J. Physiol. 269:F449-F457.

12. Tomita, K., Nonguchi, H., Terada, Y., and Marumo, F. 1993. Effects of ET-1 on water and chloride transport in cortical collecting ducts of the rat. Am. J. Physiol. 264:F690-F696.

13. Tomita, K., Nonoguchi, H., and Marumo, F. 1990. Effects of endothelin on peptide-dependent cyclic adenosine monophosphate accumulation along the nephron segments of the rat. J. Clin. Invest. 85:2014-2018.

14. Kohan, D.E., Padilla, E., and Hughes, A.K. 1993. Endothelin B receptor mediates ET-1 effects on cAMP and PGE2 accumulation in rat IMCD. Am. J. Physiol. 265:F670-F676.

15. Edwards, R.M., Stack, E.J., Pullen, M., and Nambi,
P. 1993. Endothelin inhibits vasopressin action in rat inner medullary collecting duct via the ETB receptor. J. Pharmacol. Exp. Ther. 267:1028-1033.

16. Oishi, R., Nonoguchi, H., Tomita, K., and Marumo, F. 1991. Endothelin-1 inhibits AVP-stimulated osmotic water permeability in rat medullary collecting duct. Am. J. Physiol. 261:F951-F956.

17. Nadler, S.P., Zimplemann, J.A., and Hebert, R.L. 1992. Endothelin inhibits vasopressin-stimulated water permeability in rat terminal inner medullary collecting duct. J. Clin. Invest. 90:1458-1466.

18. Ling, B.N. 1994. Luminal endothelin-1 inhibits apical $\mathrm{Na}^{+}$and $\mathrm{Cl}^{-}$channels in cultured rabbit CCT principal cells [abstract]. J. Am. Soc. Nephrol. 5:292.

19. Kurokawa, K., et al. 1993. Regulation of cortical collecting duct function: effect of endothelin. Am. Heart. J. 125:582-588.

20. Zeidel, M.L., Brady, H.R., Kone, B.C., Gullans, S.R., and Brenner, B.M. 1989. Endothelin, a peptide inhibitor of $\mathrm{Na}^{+}-\mathrm{K}^{+}$-ATPase in intact tubular epithelial cells. Am. J. Physiol. 257:C1101-C1107.

21. Denton, K.M., and Anderson, W.P. 1990. Vascular actions of endothelin in the rabbit kidney. Clin. Exp. Pharmacol. Physiol. 17:861-872.

22. Schramek, H., Willinger, C.C., Gstraunthaler, G., and Pfaller, W. 1992. Endothelin-3 modulates glomerular filtration rate in the isolated perfused rat kidney. Renal. Physiol. Biochem. 15:325-333.

23. Schnermann, J., Lorenz, J.N., Briggs, J.P., and Keiser, J.A. 1992. Induction of water diuresis by endothelin in rats. Am. J. Physiol. 263:F516-F526.

24. Kamphuis, C., Yates, N.A., and McDaugall, J.G. 1994. Differential blockade of the renal vasoconstrictor and diuretic responses to endothelin- 1 by endothelin antagonist. Clin. Exp. Pharmacol. Physiol. 21:329-333.

25. Goetz, K., et al. 1989. Endothelin and sarafotoxin produce dissimilar effects on renal blood flow, but both block the antidiuretic effects of vasopressin. Proc. Soc. Exp. Biol. Med. 191:425-427.

26. Freed, M.I., Thompson, K.A., Wilson, D.E., Etheredge, R., and Jorkasky, D.K. 1996. Endothelin receptor antagonism does not alter renal hemodynamic responses or urinary sodium excretion in healthy humans [abstract]. J. Am. Soc. Nephrol. 7:1580.

27. Baynash, A.G., et al. 1994. Interaction of endothelin-3 with endothelin-B receptor is essential for development of epidermal melanocytes and enteric neurons. Cell. 79:1277-1285.

28. Hosoda, K., et al. 1994. Targeted and natural (Piebald-Lethal) mutations of endothelin-B receptor gene produce megacolon associated with spotted coat color in mice. Cell. 80:1267-1276.

29. Kurihara, Y., et al. 1995. Aortic arch malformations and ventricular septal defect in mice deficient in endothelin-1. J. Clin. Invest. 96:293-300.

30. Kurihara, Y., et al. 1994. Elevated blood pressure and craniofacial abnormalities in mice deficient in endothelin-1. Nature. 368:703-710.

31. Puffenberger, E.G., et al. 1994. A missense mutation of the endothelin-B receptor gene in multigenic Hirschsprung's disease. Cell. 79:1257-1266.

32. Gariepy, C.E., Williams, S.C., Richardson, J.A., Hammer, R.E., and Yanagisawa, H. 1998. Transgenic expression of the endothelin-B receptor prevents congenital intestinal aganglionosis in a rat model of Hirschsprung disease. J. Clin. Invest. 102:1092-1101.

33. Gariepy, C.E., Ohuchi, T., Williams, S.C., Richardson, J.A., and Yanagisawa, M. 2000. Salt-sensitive hypertension in endothelin-B receptor-deficient rats. J. Clin. Invest. 105:925-933.

34. Gariepy, C.E., Wang, Y., Nguyen, N.D.T., Li, S., and Yanagisawa, M. 2001. Salt-sensitive hypertension in ETB-deficient rats is not due to mechanisms intrinsic to the kidney [abstract]. Presented at: 7th International Conference on Endothelin. September 16-19. Edinburgh, United Kingdom.

35. Berthiaume, N., Yanagisawa, M., and D'OrleansJuste, P. 2000. Contribution of endogenous endothelin-1 and endothelin-A-receptors to the hypertensive state of endothelin-B heterozygous $(+/-)$ knockout mice. J. Cardiovasc. Pharmacol. 36(Suppl.):S72-S74.

36. Nelson, R., Stricklett, P., Ausiello, D., Brown, D., and Kohan, D. 1998. Expression of a Cre recombinase transgene by the aquaporin-2 promoter in kidney and male reproductive system of transgenic mice. Am. J. Physiol. 275:C216-C226.

37. Stricklett, P., Nelson, R., and Kohan, D. 1998. Sitespecific recombination using an epitope-tagged bacteriophage P1 Cre recombinase. Gene. 215:415-423.

38. MacCumber, M.W., Ross, C.A., Glaser, B.M., and Snyder, S.H. 1989. Endothelin: visualization of mRNAs by in situ hybridization provides evidence for local action. Proc. Natl. Acad. Sci. U. S. A. 86:7285-7289.

39. Wilkes, B.M., et al. 1991. Localization of endothelinlike immunoreactivity in rat kidneys. Am. J. Physiol. 260:F913-F920.

40. Rubera, I., et al. 2003. Collecting duct-specific gene inactivation of $\alpha \mathrm{ENaC}$ in the mouse kidney does not impair sodium and potassium balance. J. Clin. Invest. 112:554-565. doi:10.1172/JCI200316956. 
41. Schiffrin, E.L. 2000. Endothelin: role in experimental hypertension. J. Cardiovasc. Pharmacol. 35(Suppl. 2):S33-S35.

42. Goligorsky, M.S., et al. 1991. Role of endothelin in the development of Dahl hypertension. J. Cardiovasc. Pharmacol. 17(Suppl 7):S484-S491.

43. Hughes, A.K., Cline, R.C., and Kohan, D.E. 1992. Alterations in renal endothelin production in the spontaneously hypertensive rat. Hypertension. 20:666-673.

44. Kitamura, K., et al. 1989. Immunoreactive endothelin in rat kidney inner medulla: marked decrease in spontaneously hypertensive rats. Biochem. Biophys. Res. Commun. 162:38-44.

45. Vogel, V., Backer, A., Heller, J., and Kramer, H.J. 1999. The renal endothelin system in the Prague hypertensive rat, a new model of spontaneous hypertension. Clin. Sci. 97:91-98.

46. Hoffman, A., Grossman, E., Abassi, Z.A., and Keiser, H.R. 1994. Renal endothelin and hypertension [letter]. Nature. 372:50

47. Hwang, Y.S., Hsieh, T.J., Lee, Y.J., and Tsai, J.H. 1998. Circadian rhythm of urinary endothelin1 excretion in mild hypertensive patients. Am.J. Hypertens. 11:1344-1351.

48. Zoccali, C., Leonardis, D., Parlongo, S., Mallamaci, F., and Postorino, M. 1995. Urinary and plasma endothelin 1 in essential hypertension and in hypertension secondary to renoparenchymal disease. Nephrol. Dial. Transplant. 10:1320-1323.

49. Ferri, C., et al. 1997. Elevated plasma and urinary
endothelin-I levels in human salt-sensitive hypertension. Clin. Sci. 93:35-41.

50. Gallego, M.S., and Ling, B.N. 1996. Regulation of amiloride-sensitive sodium channels by endothelin-1 in distal nephron cells. Am. J. Physiol. 271:F451-F460.

51. Berger, S., et al. 1998. Mineralocorticoid receptor knockout mice: pathophysiology of $\mathrm{Na}+$ metabolism. Proc. Natl. Acad. Sci. U. S. A. 95:9424-9429.

52. Schulz-Baldes, A., et al. 2001. Induction of the epithelial $\mathrm{Na}^{+}$channel via glucocorticoids in mineralocorticoid receptor knockout mice. Pflugers Arch. 443:297-305.

53. Dean, R., Zhuo, J., Alcorn, D., Casley, D., and Mendelsohn, F.A. 1996. Cellular localization of endothelin receptor subtypes in the rat kidney following in vitro labelling. Clin. Exp. Pharmacol. Physiol. 23:524-531.

54. Wilkes, B.M., et al. 1991. Characterization of endothelin 1 receptor and signal transduction mechanisms in rat medullary interstitial cells. Am. J. Physiol. 260:F579-F589.

55. Hughes, A.K., Barry, W.H., and Kohan, D.E. 1995. Identification of a contractile function for renal medullary interstitial cells. J. Clin. Invest. 96:411-416.

56. Zhou, J. 2000. Renomedullary interstitial cells: a target for endocrine and paracrine actions of vasoactive peptides in the renal medulla. Clin. Exp. Pharmacol. Physiol. 27:465-473.

57. Silldorff, E.P., Yang, S., and Pallone, T.L. 1994. Constriction of outer medullary descending vasa recta by endothelins: modulation by $\mathrm{PGE}_{2}$ [abstract]. J. Am. Soc. Nephrol. 5:592.

58. Brodsky, S., et al. 2000. Effects of A-192621.1, a specific endothelin-B antagonist, on intrarenal hemodynamic responses to endothelin-1. J. Cardiovasc. Pharmacol. 36(Suppl 1):S311-S313.

59. Evans, R., Madden, A., Oliver, J., and Lewis, T. 2001. Effects of ETA- and ETB-receptor antagonists on regional kidney blood flow, and response to intravenous endothelin-1, in anaesthetized rabbits. J. Hypertens. 19:1789-1799.

60. Muirhead, E. 1991. The medullipin system of blood pressure control [review]. Am. J. Hypertens. 4:556S-568S.

61. Escalante, B., McGiff, J., and Oyekan, A. 2002. Role of cytochrome P-450 arachidonate metabolites in endothelin signaling in rat proximal tubule. Am.J. Physiol. 282:F144-F150.

62. Kohan, D.E., and Padilla, E. 1993. Osmolar regulation of endothelin-1 production by rat inner medullary collecting duct. J. Clin. Invest. 91:1235-1240.

63. Srinivas, S., et al. 2002. Cre reporter strains produced by targeted insertion of EYFP and ECFP into the ROSA26 locus [abstract]. BMC Dev. Biol. 1:4.

64. Shang-jin, S., Nguyen, H., Sharma, G., Navar, L., and Pandey, K. 2001. Genetic disruption of atrial natriuretic peptide receptor-A alters renin and angiotensin II levels. Am. J. Physiol. 281:F665-F673.

65. Lantelme, P., et al. 2002. Effects of dietary sodium and genetic background on angiotensinogen and renin in mouse. Hypertension. 39:1007-1014. 